\title{
Bedside troubleshooting during venovenous extracorporeal membrane oxygenation (ECMO)
}

\author{
Bhoumesh Patel ${ }^{1}$, Michael Arcaro ${ }^{2}$, Subhasis Chatterjee ${ }^{3,4}$ \\ ${ }^{1}$ Division of Cardiovascular Anesthesiology and Critical Care, ${ }^{2}$ Division of Cardiothoracic Transplantation and Circulatory Support, Baylor \\ College of Medicine/Texas Heart Institute, CHI St. Luke's Health-Baylor St. Luke's Medical Center, Houston, TX, USA; ${ }^{3}$ Michael E. DeBakey \\ Department of Surgery, Division of General Surgery, ${ }^{4}$ Michael E. DeBakey Department of Surgery, Division of Cardiothoracic Surgery, Baylor \\ College of Medicine, Houston, TX, USA \\ Contributions: (I) Conception and design: All authors; (II) Administrative support: All authors; (III) Provision of study materials or patients: All \\ authors; (IV) Collection and assembly of data: All authors; (V) Data analysis and interpretation: All authors; (VI) Manuscript writing: All authors; (VII) \\ Final approval of manuscript: All authors. \\ Correspondence to: Subhasis Chatterjee, MD, FACS, FACC, FCCP. Department of Surgery, Divisions of General Surgery and Cardiothoracic Surgery, \\ Baylor College of Medicine, One Baylor Plaza, MS: BCM 390, Houston, TX 77030-3411, USA. Email: Subhasis.Chatterjee@bcm.edu.
}

\begin{abstract}
In this review, we discuss common difficulties that clinicians may encounter while managing patients treated with venovenous (VV) extracorporeal membrane oxygenation (ECMO). ECMO is an increasingly important tool for managing severe respiratory failure that is refractory to conventional therapies. Its overall goal is to manage respiratory failure-induced hypoxemia and hypercarbia to allow "lung rest" and promote recovery. Typically, by the time VV-ECMO is initiated, the patient's pulmonary condition requires conventional ventilator settings that are detrimental to lung recovery or that exceed the remaining functional lung's ability to maintain acceptable physiological conditions. Standard mechanical ventilation can activate inflammation and worsen the pulmonary damage caused by the underlying disease, leading to ventilator-induced lung injury. In contrast, VV-ECMO facilitates lung-protective ventilation, decreasing further ventilator-induced lung injury and allowing lung recovery. Such lung-protective ventilation seeks to avoid barotrauma (by monitoring transpulmonary pressure), volutrauma (by reducing excessive tidal volume to promote lung rest), atelectotrauma [by maintaining adequate positive end-expiratory pressure (PEEP)], and oxygen toxicity (by decreasing ventilator oxygen levels when PEEP is adequate). ECMO for adult respiratory failure was associated with overall survival of $62 \%$ in 2018, according to the Extracorporeal Life Support Organization (ELSO) January 2019 registry report. Difficulties that may arise during VVECMO require timely diagnosis and optimal management to achieve the most favorable outcomes. These difficulties include ventilation issues, hypoxemia (especially as related to recirculation or low ECMO-flowto-cardiac-output ratio), sepsis, malfunctioning critical circuit components, lack of clarity regarding optimal hemoglobin levels, hematological/anticoagulation complications, and right ventricular (RV) dysfunction. A culture of safety should be emphasized to optimize patient outcomes. A properly functioning team-not only the bedside clinician, but also nurses, perfusionists, respiratory therapists, physical therapists, pharmacists, nutritionists, and other medical specialists and allied health personnel—is vital for therapeutic success.
\end{abstract}

Keywords: Extracorporeal membrane oxygenation (ECMO); ventilator-induced lung injury; acute respiratory distress syndrome (ARDS)

Submitted Feb 26, 2019. Accepted for publication Apr 17, 2019.

doi: $10.21037 /$ jtd.2019.04.81

View this article at: http://dx.doi.org/10.21037/jtd.2019.04.81

(C) Journal of Thoracic Disease. All rights reserved. 


\section{Introduction}

Mortality from severe respiratory failure and acute respiratory distress syndrome (ARDS) ranges from 40\% to $50 \%$ with conventional medical management (1). Venovenous (VV) extracorporeal membrane oxygenation (ECMO) is evolving as a treatment option for managing severe respiratory failure that is refractory to conventional therapies. The use of ECMO continues to expand as monitoring and management methods improve. ECMO has been used successfully as a bridge to lung transplant, for primary graft dysfunction after lung transplantation, during or after high-risk thoracic operations, and for treating massive pulmonary hemorrhage and trauma (2-4). The primary goal of $\mathrm{VV}$-ECMO is to manage hypoxemia and hypercarbia due to respiratory failure, so as to allow lung rest and promote subsequent recovery.

The value of VV-ECMO in treating ARDS is difficult to fully determine solely from the number of ECMO cases as a proportion of overall severe ARDS cases. According to a January 2019 report from the Extracorporeal Life Support Organization (ELSO), most of the ECMO used for respiratory failure in adults (90-95\%) used a VV configuration; the procedure was used 19,467 times and achieved a $62 \%$ survival rate (5).

Critical to any successful ECMO program is a culture of safety, which should be promoted to optimize patient outcomes. A properly functioning team-not only the bedside clinician, but also nurses, perfusionists, respiratory therapists, physical therapists, pharmacists, nutritionists, and a range of other medical specialists and allied health personnel-is vital for therapeutic success.

In this review, we discuss common difficulties that a clinician may encounter while managing patients treated with VV-ECMO.

\section{Mechanical ventilation on ECMO}

By the time ECMO is initiated, the patient's pulmonary condition typically requires mechanical ventilator settings that are detrimental to lung recovery or that have exceeded the remaining functional lung's ability to preserve homeostasis. Standard mechanical ventilation can cause lung injury by activating inflammation that worsens pulmonary damage caused by the underlying disease. In many cases of severe ARDS, the recommended conventional therapy leads to difficulty maintaining adequate oxygenation, ventilation, and acid-base parameters. In contrast, lung- protective ventilation, such as that provided by ECMO, seeks to avoid barotrauma (by monitoring transpulmonary pressure and avoiding high airway pressures), volutrauma (by avoiding excessive tidal volumes, thereby allowing the lung to rest), atelectotrauma [by maintaining adequate positive end-expiratory pressure (PEEP)], and oxygen toxicity (by decreasing ventilator oxygen levels when PEEP is adequate) (6). Maintaining a low driving pressure (inspiratory plateau pressure - PEEP) has been associated with improved survival in patients with ARDS (7).

Consensus is lacking on the ideal baseline ventilator settings for VV-ECMO. Typically, the initial circuit $\mathrm{FIO}_{2}$ (fraction of inspired oxygen) is set at 1.0 with a sweep gasflow rate of $2-5 \mathrm{~L} / \mathrm{min}$ and the revolutions per minute (RPM) set to achieve $50-80 \mathrm{~mL} / \mathrm{kg} / \mathrm{min}$ of flow. Arterial blood gas should be normalized over several hours rather than quickly after ECMO initiation, because of cerebral edema concerns. In one study of patients receiving ECMO, the only ventilator parameter that was independently associated with reduced mortality while on ECMO was minimizing driving pressure (8). Another review suggested that a target tidal volume of $4 \mathrm{~mL} / \mathrm{kg}$ of predicted body weight (ultra-lung-protective ventilation) may have better outcomes than the standard $6 \mathrm{~mL} / \mathrm{kg}$ (9). Currently, the recommended ventilation strategy with ECMO is reducing tidal volume to $4-6 \mathrm{~mL} / \mathrm{kg}$ of predicted body weight and maintaining a plateau pressure of $\leq 25 \mathrm{cmH}_{2} \mathrm{O}$, with a PEEP of $10 \mathrm{cmH}_{2} \mathrm{O}$ (6). In addition, the goal should be the weaning of neuromuscular blockade and pulmonary vasodilators while achieving these lung-protective ventilation settings within 12-24 hours.

\section{Hypoxemia}

The ability of human beings to tolerate extreme hypoxemia was illustrated among mountain climbers at Mount Everest, who were found to tolerate a mean $\mathrm{SaO}_{2}$ (arterial oxygen saturation) of $54 \%$ and a $\mathrm{PaO}_{2}$ (partial pressure of oxygen) of $25 \mathrm{mmHg}$ (10). Although these levels might be tolerable for healthy individuals, it is not yet known whether these results offer meaningful insight into ARDS patient management. Moreover, there is no consensus on the minimum acceptable $\mathrm{SaO}_{2}$ level during $\mathrm{VV}$-ECMO. ELSO guidelines recommend a target $\mathrm{SaO}_{2}$ of $80-85 \%$ at rest ventilator settings, so long as cardiac output and hemoglobin levels are adequate (11); conversely, the Columbia group (12) advises a target $\mathrm{SaO}_{2}>88 \%$. In one review of ELSO registry data from 2010-2015, Munshi 


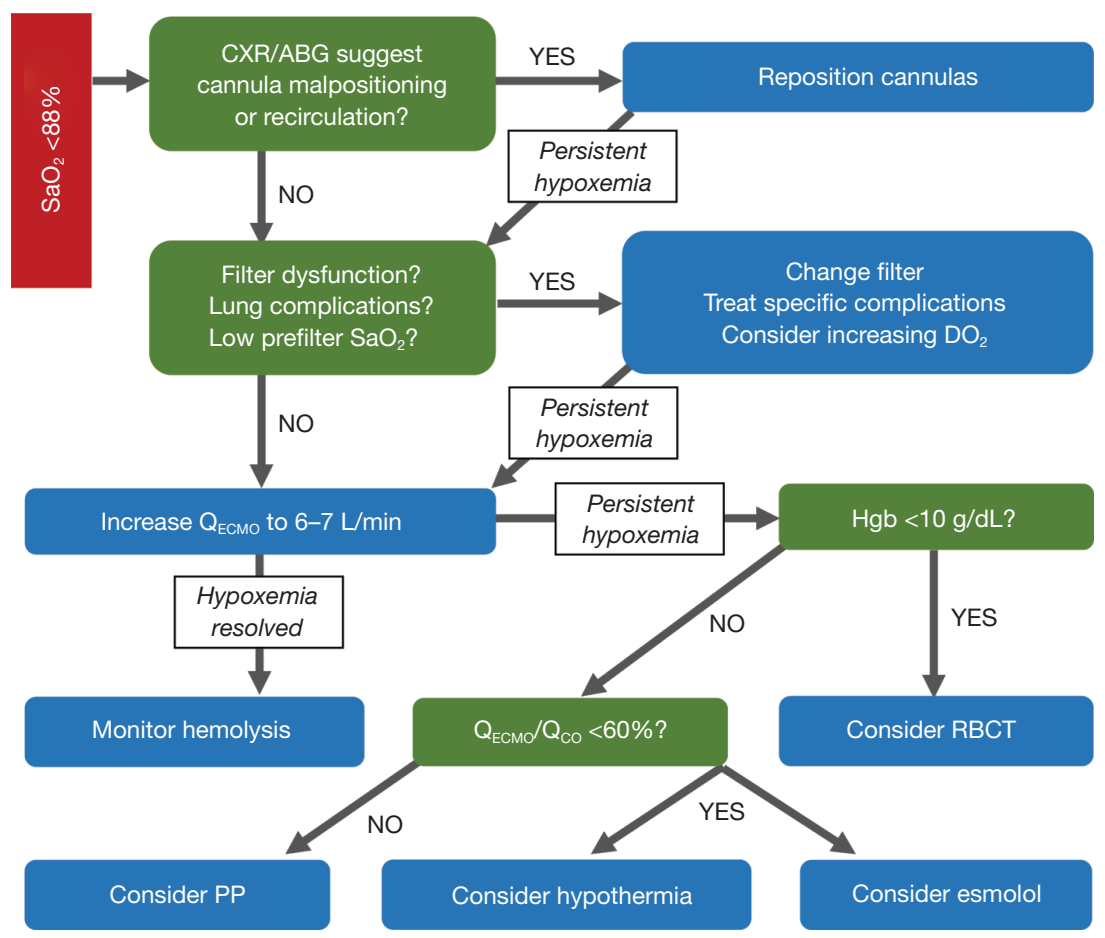

Figure 1 Diagnostic and management algorithm for addressing hypoxemia on venovenous ECMO. Adapted by permission from (14). $\mathrm{SaO}_{2}$, arterial oxygen saturation; CXR, chest X-rays; ABG, arterial blood gases; $\mathrm{DO}_{2}$, oxygen delivery; $\mathrm{Q}_{\mathrm{ECMO}}$, extracorporeal membrane oxygenation (ECMO) blood flow; Hgb, hemoglobin; $\mathrm{Q}_{\mathrm{CO}}$, cardiac output; RBCT, red blood cell transfusion; PP, prone positioning.

and colleagues (13) analyzed results from 1,952 patients, of whom 765 had undergone VV-ECMO. In the VVECMO cohort (mean age, 44 years), the median duration of mechanical ventilation was 64 hours before ECMO initiation, and the median duration of ECMO support was 8 days. After 24 hours of ECMO support, patients who achieved normoxemia $\left(\mathrm{PaO}_{2}\right.$ of $\left.61-100 \mathrm{mmHg}\right)$ were compared with those with sustained hypoxemia $\left(\mathrm{PaO}_{2}<60 \mathrm{mmHg}\right)$ or moderate hyperoxemia $\left(\mathrm{PaO}_{2}\right.$ of $101-300 \mathrm{mmHg}$ ). Mortality was similarly elevated in the moderate hyperoxemia group [odds ratio (OR): 1.66; $95 \%$ confidence interval (CI): 1.11 to 2.50$]$ and the hypoxemia group (OR: $1.68 ; 95 \%$ CI: 1.09 to 2.57 ), compared with the normoxemia group. This review suggests that a higher targeted oxygen level resulting in moderate hyperoxemia might not be beneficial and may actually be as harmful as persistent hypoxemia. This result is hypothesis-generating and offers a prospective target for future investigation.

In VV-ECMO, the crucial determinants of oxygen saturation are the oxygen fraction of the circuit, the ratio of ECMO flow to native cardiac output, metabolic demand, native lung function, and recirculation. Recirculation occurs when reinjected oxygenated blood that is intended to cross the tricuspid valve into the pulmonary circulation is instead withdrawn by the venous drainage cannula without passing through its systemic circulation. Recirculation and a low ECMO-flow-to-cardiac-output ratio are two common causes of persistent hypoxemia with ECMO flow. For patients with an oxygen saturation $<85 \%$, we suggest the stepwise approach adapted from Levy et al. (14) (Figure 1). It is important to distinguish between hypoxemia, or low oxygen content in the blood $\left(\mathrm{SaO}_{2}\right)$, and hypoxia, or low oxygen content in the tissue. Whereas hypoxemia can be easily measured, tissue hypoxia (which can only be measured indirectly, such as with serum lactate levels) determines whether the body is forced into anaerobic metabolism and incurs its deleterious effects.

\section{Recirculation}

The classic signs of recirculation are low $\mathrm{SaO}_{2}$ and high $\mathrm{SpreO}_{2}$ (preoxygenator saturation). Some initial improvement in $\mathrm{SaO}_{2}$ occurs with increased pump flow; however, increasing the circuit flow will eventually increase 

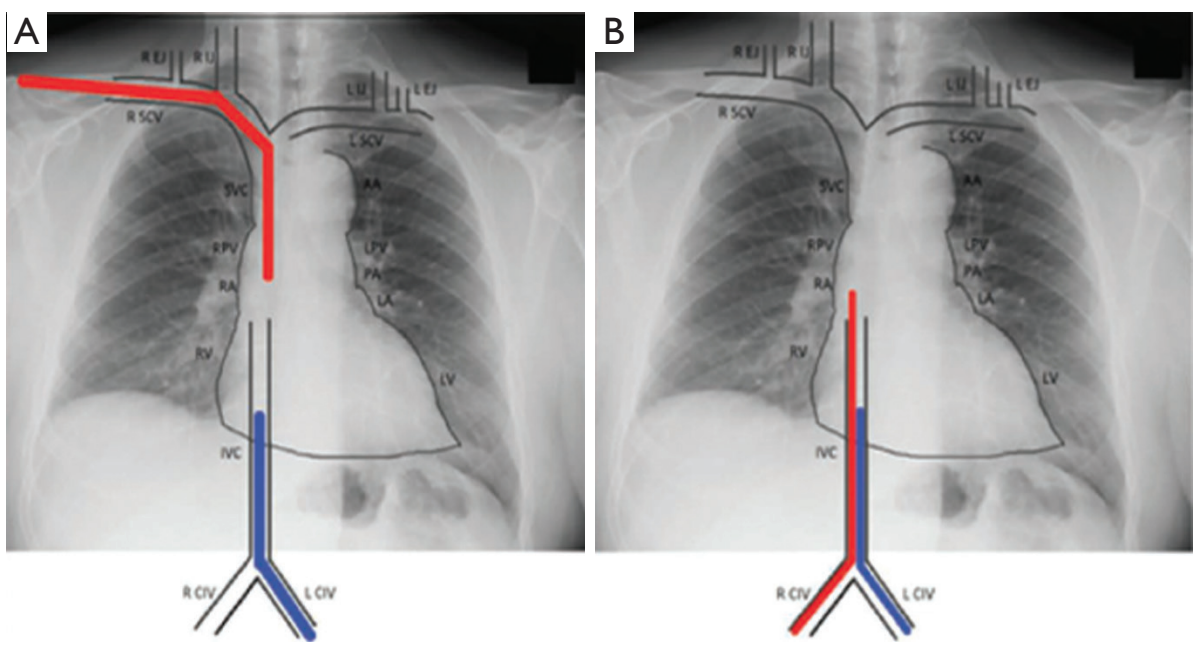

Figure 2 Optimal cannula positioning in femoral/jugular configuration (A) and femoral/femoral configuration (B). R/L, right/left; CFV, common femoral vein; IVC, inferior vena cava; RA/LA, right/left atrium; RV/LV, right/left ventricle; SVC, superior vena cava; R/L PV, right/left pulmonary vein; R/L IJV, right/left internal jugular vein; R/L SCV, right/left subclavian vein.

recirculation and reduce the effective blood flow (15). Although no single calculation method has been shown to accurately predict recirculation, we recommend the following formula to quantify recirculation when needed:

Recirculation $(\%)=\left(\mathrm{SpreO}_{2}-\mathrm{SvO}_{2}\right) /\left(\mathrm{SpostO}_{2}-\mathrm{SvO}_{2}\right)$ $\times 100$

In this formula, $\mathrm{SpreO}_{2}$ is the saturation of blood entering the oxygenator, $\mathrm{Spost}_{2}$ is the saturation of blood leaving the oxygenator, and $\mathrm{SvO}_{2}$ is the saturation of venous blood returning to the venae cavae just before being drained by the ECMO circuit. If the $\mathrm{SpreO}_{2}$ is the same as the $\mathrm{SvO}_{2}$, there is $0 \%$ recirculation. If the $\mathrm{SpreO}_{2}$ is the same as the $\mathrm{SpostO}_{2}$, there is $100 \%$ recirculation. Typically, if the $\mathrm{SpreO}_{2}$ is $<75 \%$, clinically significant recirculation is unlikely.

Factors that can affect the degree of recirculation include the pump speed, blood flow rates, direction of extracorporeal flow, and the cannula type, size, and position. Once a high recirculation percentage is diagnosed, the following steps should be taken to decrease recirculation:

(I) for patients receiving two-site VV-ECMO, increase the distance between the drainage and reinfusion ports, with a target separation distance of approximately $15 \mathrm{~cm}$ (Figure 2);

(II) add a drainage cannula to achieve a similar, effective pump flow rate at a lower speed;

(III) switch to a bicaval, dual-lumen cannula under echocardiographic and fluoroscopic guidance, and then direct the reinfusion jet toward the tricuspid valve.

\section{Ratio of ECMO flow to cardiac output}

With typical ECMO flows of 3-4 L/min, some of the blood flows through the ECMO circuit and some is shunted past the ECMO circuit into the diseased native lung. This leads to a mixing of well-oxygenated ECMO blood with poorly oxygenated shunted blood, which lowers total oxygen saturation $\left(\mathrm{SaO}_{2}\right)$. In the absence of high recirculation, an ECMO-flow-to-cardiac-output ratio $>60 \%$ has been shown to maintain adequate blood oxygenation (16). Increasing ECMO flow is a reasonable initial maneuver when initially confronting hypoxemia. However, excessive native cardiac output in relation to the ECMO flow can exacerbate hypoxemia by creating an imbalance in that ratio. Strategies to reduce cardiac output include avoiding hyperthermia and providing adequate sedation and analgesia. If hypoxemia and high cardiac output persist, esmolol administration or hypothermia can be initiated, as the patient tolerates.

\section{Circuit issues}

Any of the critical components of the circuit (cannulas, pump, tubing, oxygenator; Figure 3) can malfunction. Constant observation and inspection are crucial to maintaining circuit integrity and patient safety. Routinely 


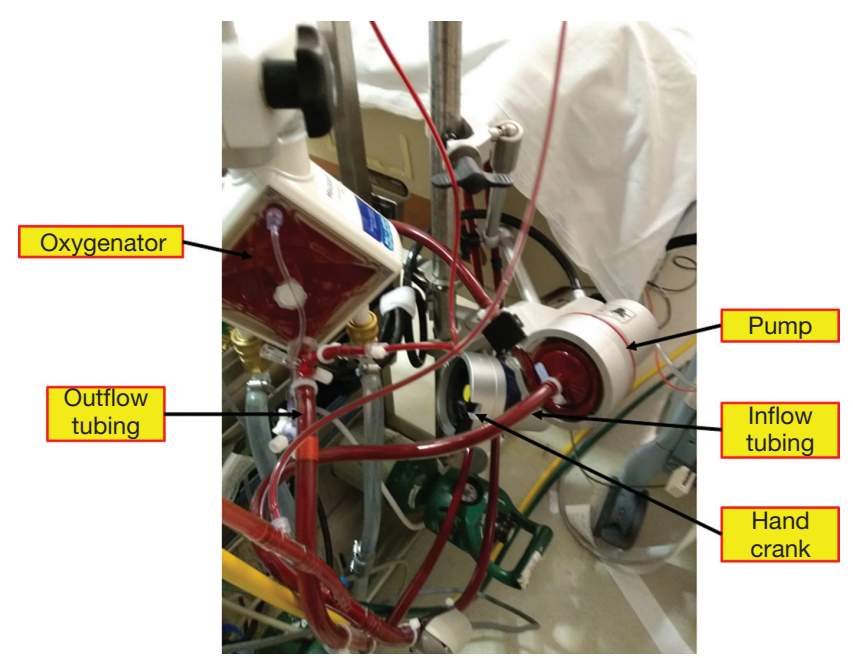

Figure 3 ECMO cannula circuit and components. ECMO, extracorporeal membrane oxygenation.

inspecting all circuit components and tracing gas flow to the oxygenator should detect most problems before clinically meaningful events occur. Close communication among physician, perfusionist, and nurse is necessary for optimal management.

Decreased circuit flows can be caused by various factors, including hypovolemia, malpositioned cannulas, tubing kinks, circuit obstructions, and compromised hemodynamic status. Impaired venous drainage commonly manifests as "chattering" (i.e., visible vibration or shaking within the venous line). Chattering occurs when extremely low negative pressure around the drainage port causes the vessel to collapse around the cannula. As negative pressure builds back up, the vessel releases and begins to chatter. Centrifugal pumps can increase negative pressure $(600 \mathrm{mmHg}$ of suction), which not only decreases blood flow, but also can induce cavitation and hemolysis. If chattering occurs, the RPM should be reduced to achieve a more consistent flow by alleviating the negative pressure. If the chattering is caused by hypovolemia, the addition of crystalloid typically resolves the problem.

Adequate anticoagulation is needed to achieve optimal ECMO circuit longevity; however, complications often prevent this from happening, placing the circuit at risk. If adequate anticoagulation cannot be achieved, high flows (4-5 L/min) are recommended to minimize stagnation and clot formation within the circuit (17). Visual inspection can reveal clot formation around cannulas, within the centrifugal cone, or on the oxygenator. Consider replacing the circuit if clot formation is hindering pump flow or oxygenation or is putting the patient at risk for embolism. If a clot cannot easily be visualized, pressure readings from the circuit can identify the affected area.

Excessive hemolysis can result from a cannula that is too small, improper cannula positioning, high RPMs, or clot formation in the centrifugal cone or within the oxygenator. Lactate dehydrogenase levels can indicate circuit-induced hemolysis, but only if sepsis is not present. Routine testing for free plasma hemoglobin can more accurately determine whether hemolysis is occurring in the circuit (18).

Oxygenator failure can result from oxygen supply failure or thrombus accumulation within the oxygenator, decreasing oxygen transfer. Ideal ECMO oxygenators are made from polymethylpentene, which causes less hemolysis, pressure drop, and plasma leakage and has a longer lifespan than materials used in earlier oxygenators $(19,20)$. A venous saturation or $\mathrm{O}_{2}$ monitoring device in the circuit can indicate whether oxygen flow has decreased or stopped. In addition, if, on visual inspection, the blood entering and exiting the oxygenator does not appear as it normally should (dark red going in, bright red coming out), this may signal problems. Backup portable oxygen tanks should either be connected directly to the ECMO circuit or be placed in the patient's room until the oxygen supply issue is resolved. Arterial blood gas levels and membrane pressures, if applicable, can be measured before and after the blood passes through the oxygenator to determine whether the oxygenator is running at full capacity. A normal pressure drop across a membrane is $<50 \mathrm{mmHg}$; a change in pressure $>100 \mathrm{mmHg}$ strongly suggests an obstruction within the oxygenator (21). A failing oxygenator should be replaced to optimize oxygenation and reduce the risk of thrombus embolization. Accomplishing this efficiently in an acute situation requires that appropriate personnel be available and that the sequence of action be clearly articulated and well-rehearsed.

Catastrophic circuit issues include substantial blood loss, massive air embolism, and complete loss of pump flow. In the event of a catastrophic incident, the patient should first be isolated from the circuit by clamping both venous and arterial ECMO lines as close to the patient as possible to prevent retrograde flow and possible migration and delivery of air back into the patient, or even exsanguination. Once the patient is isolated from the circuit, the problem can be identified and the circuit de-aired or exchanged, after which ECMO can be promptly resumed. Pump failure is extremely rare but can happen because of power loss, clot formation 
within the centrifugal cone, or decoupling of the centrifugal pump. A hand crank or backup pump should be attached to the ECMO circuit to maintain flow while the primary pump is being repaired or a backup pump is retrieved (Figure 3). Again, periodic simulation of catastrophic events can train the ECMO team to perform the necessary steps calmly and efficiently when these situations arise.

Because VV-ECMO supports only the pulmonary system, if cardiac dysrhythmias or cardiac arrest occurs, the normal code response should be initiated. ECMO flow will diminish if the heart is not pumping and cannot be restored until cardioversion or chest compressions take place. Converting from the ventilator to hand-bagging is also recommended, in case loss of ECMO flow occurs in the hyperdynamic state. If cardioversion is not successful, conversion to venoarterial (VA)-ECMO can be initiated.

\section{Optimal hemoglobin level}

For patients with severe ARDS treated with VV-ECMO, the optimal hemoglobin ( $\mathrm{Hgb}$ ) level has not been well studied or defined. Critically ill patients had no worse survival if they were treated with a restrictive transfusion strategy $(\mathrm{Hgb}>7 \mathrm{~g} / \mathrm{dL})$ than if they were treated with a liberal strategy $(\mathrm{Hgb}>10 \mathrm{~g} / \mathrm{dL})(22)$. However, ELSO recommends maintaining a normal hematocrit level during ECMO treatment to optimize oxygen delivery (11). Voelker et al. (23) used a hemoglobin transfusion threshold of $7 \mathrm{~g} / \mathrm{dL}$ in a group of 18 patients with ARDS and noted a $61 \%$ survival rate. Agerstrand et al. (24) used a similar transfusion threshold of $7 \mathrm{~g} / \mathrm{dL}$ in 38 patients with ARDS and noted a $74 \%$ survival rate. These results suggest that if the patient has signs of adequate perfusion, such as satisfactory $\mathrm{SvO}_{2}$ and lactate levels, then a liberal transfusion threshold may not be beneficial. A randomized trial of liberal versus conservative transfusion thresholds in ECMO may offer further insights into not only survival, but also secondary endpoints such as end-organ function and length of stay.

\section{Sepsis}

Sepsis is the most common cause of increased cardiac output and oxygen extraction (21). Ventilator-associated pneumonia and bacteremia are the most common causes of sepsis in patients on ECMO, who are at high risk for nosocomial infections (25). Because ECMO patients with sepsis rarely have fever-a result of temperature regulation by the ECMO circuit-clinical signs such as hemodynamic instability, increased fluid requirement, and decreasing arterial oxygenation can serve as signals of a new infection. A sepsis workup, including inspection of white blood cell count and microbiological analysis of blood, sputum, and urine samples, should be promptly initiated. Early, aggressive treatment is essential, with broadspectrum antibiotics and vasopressors given as needed to maintain adequate perfusion. Persistent hypoxemia due to sepsis can be improved by decreasing the metabolic rate with sedation, paralysis, or active cooling to avoid hyperthermia. Decreasing cardiac output with esmolol infusion or increasing circuit flow as tolerated should also be considered.

\section{Hematological and anticoagulation issues}

Bleeding is one of the most common complications in patients on VV-ECMO. A systematic review of 18 studies and 646 patients (26) reported bleeding in approximately $16 \%$ of cases. Anticoagulation should be monitored with tests of activated partial thromboplastin time (aPTT), antifactor $\mathrm{Xa}(\mathrm{aXa})$ assay, tests of antithrombin III levels, and thromboelastography (TEG).

Historically, the targeted aPTT has been $60-80$ seconds or 1.5-2.5 times the normal level. More recently, some authors have described using a lower target aPTT, notably in the EOLIA (ECMO to Rescue Lung Injury in Severe ARDS) study (27), in which the target aPTT was 40-55 seconds. An increasing need for heparin should raise suspicion of heparin resistance and should prompt a check of the antithrombin III level, which can be maintained within the normal range $(80-120 \%)$ with fresh-frozen plasma or concentrate. In our current practice, we begin with the aPTT (goal 50-70 s), then assess the TEG (goal R time prolongation of 2-3 times normal) and aXa heparin activity (goal $0.3-0.7 \mathrm{IU} / \mathrm{mL}$ ). Although the aPTT is the primary reference, we aim to see concordance between at least 2 of the 3 monitoring lab values.

Bleeding and thrombosis in combination suggests either heparin-induced thrombocytopenia/thrombosis or disseminated intravascular coagulation (21). In the presence of positive antibodies or strong clinical suspicion, heparin should be switched to either bivalirudin or argatroban. Heparin-coating the ECMO circuit does not appear to cause or perpetuate thrombocytopenia or thrombosis (28). The circuit should be exchanged only if thrombosis impairs gas exchange or increases resistance. Disseminated 
intravascular coagulation is usually triggered by sepsis or circuit thrombus and should be suspected in patients with an elevated aPTT, elevated prothrombin time, low fibrinogen, elevated D-dimer, or increased fibrinolysis (28). The ECMO circuit should be exchanged if a thrombus is causing disseminated intravascular coagulation; conversely, if sepsis is the cause, aggressive treatment with broadspectrum antibiotics and source control is indicated.

Increased fibrinolysis should be evaluated with viscoelastic tests, such as TEG or rotational thromboelastometry. Epsilon-aminocaproic acid may be useful for controlling bleeding and stabilizing clot formation (29). Although the optimal dosing strategy for epsilon-aminocaproic acid in ECMO patients has not been established, it is a reasonable adjunct in those with bleeding.

In our practice, platelet count and PT/international normalized ratio (INR)/aPTT are checked daily. We aim to maintain hemoglobin levels above $8 \mathrm{~g} / \mathrm{dL}$, a platelet count above 50,000, and correct the PT/INR or fibrinogen levels if there is clinical evidence of bleeding or anticipated surgical procedure.

There are published reports of managing VV-ECMO without any anticoagulation for a mean of 14 days in a series of 8 patients after cardiac surgery (30), 3 patients with ARDS after traumatic brain injury (31), and for 25 days in 1 patient with Goodpasture syndrome (32). Patients with bleeding complications may need a period of time off anticoagulation. In our practice, this is reasonably well tolerated provided that flows are at least $3.5 \mathrm{~L} / \mathrm{min}$.

\section{Right ventricular (RV) dysfunction}

In respiratory failure, hypoxemia, hypercarbia, and acidemia cause pulmonary vasoconstriction that can lead to $\mathrm{RV}$ dysfunction. This dysfunction may be ameliorated by initiating VV-ECMO to normalize $\mathrm{O}_{2}, \mathrm{CO}_{2}$, and $\mathrm{pH}$ levels. The incidence of $\mathrm{RV}$ failure related to ARDS has been reported to be $10-25 \%$ (33). Initial management should be with pulmonary vasodilators, inotropes, and diuretics. If $\mathrm{RV}$ failure persists with evidence of hypoperfusion, VV- or VAECMO should be considered, on the basis of the severity of $\mathrm{RV}$ dysfunction when cannulation is contemplated. Although the temptation will be to proceed directly to VAECMO, most RV dysfunction can be managed successfully with VV-ECMO, which typically produces fewer complications. Indeed, even patients taking two or three vasoactive medications when ECMO is initiated will have better survival outcomes with VV-ECMO than with VA-
ECMO (34).

However, for VV-ECMO patients who have persistent $\mathrm{RV}$ dysfunction, conversion to a venoarterial-venous (VAV) mode of ECMO by adding an arterial cannula is necessary to unload the right ventricle. The RV volume unloading in VA-ECMO is superior to that in VAV-ECMO; however, VAV-ECMO should be considered in patients with severe pulmonary failure and hypoxemia, because peripheral VA-ECMO may not deliver enough oxygen to the upper body (Harlequin syndrome) if left ventricular function is well-preserved $(35,36)$. When VA-ECMO is desired, central cannulation via the axillary or subclavian artery is a reasonable technique that avoids sternotomy. Alternatively, a conduit can be sewn to the ascending aorta through a limited right lateral thoracotomy.

\section{Patient positioning}

Among patients with ARDS, prone positioning has been shown to significantly improve survival rates (37). Prone positioning reduces collapse of dorsal lung segments and alveolar over-distension in ventral lung segments, optimizes lung recruitment, and enhances drainage. Indeed, some authors have argued that bypassing prone positioning for ECMO is unwarranted (38). In the EOLIA study, 56\% of the ECMO patients had been positioned prone before ECMO was initiated. Several case series have shown the feasibility and safety of prone positioning during ECMO (39-41). For patients on VV-ECMO, prone positioning can be performed safely when carried out by a skilled and well-trained team. Prone positioning in VV-ECMO may be worthy of further investigation in randomized trials performed at experienced centers.

In a recent ELSO International Summary of data regarding adult ECMO for respiratory failure (11), of the almost 14,000 ECMO runs from 2014-2018, VV-ECMO was used in $88.6 \%$, VA-ECMO in $8.4 \%$, and VAV-ECMO in $1.9 \%$. The average VV-ECMO run was 292 hours (12.2 days), with $61 \%$ survival. The longest reported run during that period was 315.6 days.

The general principles behind weaning from VV-ECMO include being able to resume lung-protective ventilation with adequate oxygenation and ventilation. If the underlying disease proves treatable, and if chest radiographic and arterial blood gas findings improve, weaning from ECMO is probably achievable. Assessment for weaning should be performed daily. As carbon dioxide clearance improves, the sweep gas flow is reduced and may be weaned off completely 
for several hours or overnight before decannulation. As oxygenation improves, ECMO flow rates can be reduced to $2 \mathrm{~L} / \mathrm{min}$, and circuit $\mathrm{FIO}_{2}$ can be reduced from 1.0 to lower levels (0.21 to 0.4$)$. During this transition, the ventilator assumes a greater share of the respiratory support away from the ECMO circuit. If a tracheostomy is planned and a prolonged recovery is anticipated, the tracheostomy is performed while the patient is still on ECMO, which can reduce sedation requirements after decannulation and improve mobility afterwards $(42,43)$.

\section{Conclusions}

VV-ECMO is an increasingly important tool for managing severe ARDS. Common situations and problems that may arise during ECMO require timely diagnosis and optimal management to achieve the most favorable therapeutic outcomes.

\section{Acknowledgments}

JF Woodruff, BS, ELS, and SN Palmer, PhD, ELS, contributed to the editing of the manuscript.

\section{Footnote}

Conflicts of Interest: The authors have no conflicts of interest to declare.

\section{References}

1. Ranieri VM, Rubenfeld GD, Thompson BT, et al. Acute respiratory distress syndrome: the Berlin Definition. JAMA 2012;307:2526-33.

2. Bosarge PL, Raff LA, McGwin G Jr, et al. Early initiation of extracorporeal membrane oxygenation improves survival in adult trauma patients with severe adult respiratory distress syndrome. J Trauma Acute Care Surg 2016;81:236-43.

3. Lang G, Taghavi S, Aigner C, et al. Extracorporeal membrane oxygenation support for resection of locally advanced thoracic tumors. Ann Thorac Surg 2011;92:264-70.

4. Mattar A, Chatterjee S, Loor G. Bridging to lung transplantation. Crit Care Clin 2019;35:11-25.

5. Extracorporeal Life Support Organization. ECLS Registry Report International Summary. January 2019 [cited February 7, 2019]. Available online: https://www.
elso.org/Portals/0/Files/Reports/2019/International\%20 Summary\%20January\%202019.pdf

6. Schmidt M, Pellegrino V, Combes A, et al. Mechanical ventilation during extracorporeal membrane oxygenation. Crit Care 2014;18:203.

7. Amato MB, Meade MO, Slutsky AS, et al. Driving pressure and survival in the acute respiratory distress syndrome. $\mathrm{N}$ Engl J Med 2015;372:747-55.

8. Serpa Neto A, Schmidt M, Azevedo LC, et al. Associations between ventilator settings during extracorporeal membrane oxygenation for refractory hypoxemia and outcome in patients with acute respiratory distress syndrome: a pooled individual patient data analysis. Intensive Care Med 2016;42:1672-84.

9. Marhong JD, Munshi L, Detsky M, et al. Mechanical ventilation during extracorporeal life support (ECLS): a systematic review. Intensive Care Med 2015;41:994-1003.

10. Grocott MP, Martin DS, Levett DZ, et al. Arterial blood gases and oxygen content in climbers on Mount Everest. N Engl J Med 2009;360:140-9.

11. Extracorporeal Life Support Organization. Extracorporeal Life Support Organization (ELSO) guidelines for adult respiratory failure. August 2017 [cited February 7, 2019]. Available online: https://www.elso.org/ Portals/0/ELSO\%20Guidelines\%20For\%20Adult\%20 Respiratory\%20Failure\%201_4.pdf

12. Brodie D, Bacchetta M. Extracorporeal membrane oxygenation for ARDS in adults. N Engl J Med 2011;365:1905-14.

13. Munshi L, Kiss A, Cypel M, et al. Oxygen thresholds and mortality during extracorporeal life support in adult patients. Crit Care Med 2017;45:1997-2005.

14. Levy B, Taccone FS, Guarracino F. Recent developments in the management of persistent hypoxemia under venovenous ECMO. Intensive Care Med 2015;41:508-10.

15. Abrams D, Bacchetta M, Brodie D. Recirculation in venovenous extracorporeal membrane oxygenation. ASAIO J 2015;61:115-21.

16. Schmidt M, Tachon G, Devilliers C, et al. Blood oxygenation and decarboxylation determinants during venovenous $\mathrm{ECMO}$ for respiratory failure in adults. Intensive Care Med 2013;39:838-46.

17. Bharat A, DeCamp MM. Veno-arterial extracorporeal membrane oxygenation without therapeutic anticoagulation for intra-operative cardiopulmonary support during lung transplantation. J Thorac Dis 2017;9:E629-31.

18. Lehle K, Philipp A, Zeman F, et al. Technical-induced 
hemolysis in patients with respiratory failure supported with veno-venous ECMO - prevalence and risk factors. PloS One 2015;10:e0143527.

19. Lequier L, Horton SB, McMullan DM, et al. Extracorporeal membrane oxygenation circuitry. Pediatr Crit Care Med 2013;14:S7-12.

20. Ündar A, Wang S, Özyüksel A, et al. Pediatric devices. In: Gregory SD, Stevens MC, Fraser JF, editors. Mechanical Circulatory and Respiratory Support. London: Academic Press, 2018;271-97.

21. Sidebotham D. Troubleshooting adult ECMO. J Extra Corpor Technol 2011;43:P27-32.

22. Hébert PC, Wells G, Blajchman MA, et al. A multicenter, randomized, controlled clinical trial of transfusion requirements in critical care. N Engl J Med 1999;340:409-17.

23. Voelker MT, Busch T, Bercker S, et al. Restrictive transfusion practice during extracorporeal membrane oxygenation therapy for severe acute respiratory distress syndrome. Artif Organs 2015;39:374-8.

24. Agerstrand CL, Burkart KM, Abrams DC, et al. Blood conservation in extracorporeal membrane oxygenation for acute respiratory distress syndrome. Ann Thorac Surg 2015;99:590-5.

25. Sun HY, Ko WJ, Tsai PR, et al. Infections occurring during extracorporeal membrane oxygenation use in adult patients. J Thorac Cardiovasc Surg 2010;140:1125-32.e2.

26. Sklar MC, Sy E, Lequier L, et al. Anticoagulation practices during venovenous extracorporeal membrane oxygenation for respiratory failure. A systematic review. Ann Am Thorac Soc 2016;13:2242-50.

27. Combes A, Hajage D, Capellier G, et al. Extracorporeal membrane oxygenation for severe acute respiratory distress syndrome. N Engl J Med 2018;378:1965-75.

28. Koster A, Sänger S, Hansen R, et al. Prevalence and persistence of heparin/platelet factor 4 antibodies in patients with heparin coated and noncoated ventricular assist devices. ASAIO J 2000;46:319-22.

29. Buckley LF, Reardon DP, Camp PC, et al. Aminocaproic acid for the management of bleeding in patients on extracorporeal membrane oxygenation: Four adult case reports and a review of the literature. Heart Lung 2016;45:232-6.

30. Takagaki M, Yamaguchi H, Ikeda N, et al. Postcardiotomy venovenous extracorporeal membrane oxygenation without heparinization. Gen Thorac Cardiovasc Surg 2018. [Epub ahead of print].

31. Muellenbach RM, Kredel M, Kunze E, et al. Prolonged heparin-free extracorporeal membrane oxygenation in multiple injured acute respiratory distress syndrome patients with traumatic brain injury. J Trauma Acute Care Surg 2012;72:1444-7.

32. Herbert DG, Buscher H, Nair P. Prolonged venovenous extracorporeal membrane oxygenation without anticoagulation: a case of Goodpasture syndrome-related pulmonary haemorrhage. Crit Care Resusc 2014;16:69-72.

33. Osman D, Monnet X, Castelain V, et al. Incidence and prognostic value of right ventricular failure in acute respiratory distress syndrome. Intensive Care Med 2009;35:69-76.

34. Kon ZN, Bittle GJ, Pasrija C, et al. Venovenous versus venoarterial extracorporeal membrane oxygenation for adult patients with acute respiratory distress syndrome requiring precannulation hemodynamic support: A review of the ELSO registry. Ann Thorac Surg 2017;104:645-9.

35. Bunge JJH, Caliskan K, Gommers D, et al. Right ventricular dysfunction during acute respiratory distress syndrome and veno-venous extracorporeal membrane oxygenation. J Thorac Dis 2018;10:S674-82 .

36. Lee SH, Jung JS, Chung JH, et al. Right heart failure during veno-venous extracorporeal membrane oxygenation for H1N1 induced acute respiratory distress syndrome: Case report and literature review. Korean J Thorac Cardiovasc Surg 2015;48:289-93.

37. Guérin C, Reignier J, Richard JC, et al. Prone positioning in severe acute respiratory distress syndrome. $\mathrm{N}$ Engl J Med 2013;368:2159-68.

38. Li X, Scales DC, Kavanagh BP. Unproven and expensive before proven and cheap: Extracorporeal membrane oxygenation versus prone position in acute respiratory distress syndrome. Am J Respir Crit Care Med 2018;197:991-3.

39. Culbreth RE, Goodfellow LT. Complications of prone positioning during extracorporeal membrane oxygenation for respiratory failure: A systematic review. Respir Care 2016;61:249-54.

40. Kimmoun A, Roche S, Bridey C, et al. Prolonged prone positioning under $\mathrm{VV}$-ECMO is safe and improves oxygenation and respiratory compliance. Ann Intensive Care 2015;5:35.

41. Lucchini A, De Felippis C, Pelucchi G, et al. Application of prone position in hypoxaemic patients supported by veno-venous ECMO. Intensive Crit Care Nurs 2018;48:61-8.

42. Broman LM, Malfertheiner MV, Montisci A, et al. 
Weaning from veno-venous extracorporeal membrane oxygenation: how I do it. J Thorac Dis 2018;10:S692-7.

43. Grant AA, Hart VJ, Lineen EB, et al. A weaning protocol

Cite this article as: Patel B, Arcaro M, Chatterjee S. Bedside troubleshooting during venovenous extracorporeal membrane oxygenation (ECMO). J Thorac Dis 2019;11(Suppl 14):S1698S1707. doi: $10.21037 /$ jtd.2019.04.81 for venovenous extracorporeal membrane oxygenation with a review of the literature. Artif Organs 2018;42:605-10. 